\title{
Behavioural aspects and psychiatric findings in Noonan's syndrome
}

\author{
A Wood, A Massarano, M Super, R Harrington
}

\begin{abstract}
This study describes the behavioural phenotype and psychiatric symptoms of 21 children with Noonan's syndrome attending a paediatric genetics clinic. Data from the behavioural phenotypes questionnaire are presented that suggest that children with Noonan's syndrome tend to be clumsy, stubborn, irritable, have communication difficulties, and are 'faddy eaters'. Nearly $50 \%$ of the patients with Noonan's syndrome were psychiatric 'cases' as assessed by a standardised measure.
\end{abstract}

(Arch Dis Child 1995; 72: 153-155)

Keywords: Noonan's syndrome, behavioural phenotype, psychiatric symptoms.

Noonan's syndrome is a genetic syndrome featuring cardiac abnormalities (chiefly pulmonary stenosis), facial abnormalities, short stature, and learning difficulties. Although familial recurrence is consistent with an autosomal dominant mode of inheritance, sporadic cases occur. There is variability of expression with some transmitting family members having minimal features. ${ }^{1}$ The overall incidence is believed to be between one in 1000 and one in $2500 .^{2}$ There are few data on cognition and intelligence in Noonan's syndrome. A study by Money and Kalus of eight males with Noonan's syndrome aged 13-36 years, showed an IQ range from 64-127 with a median of $102 .^{3}$ Five of these patients showed specific verbal or praxic disparity.

There is an increasing body of knowledge about behavioural phenotypes of different genetic syndromes. ${ }^{4}$ The impetus for this research is derived from parent groups, who often cite their children's behaviour as the least understood and most difficult to cope with aspect of their genetic condition. It has been observed that children with the same genetic disorder may behave in similar ways. The term 'behavioural phenotype' should be reserved for behaviours which are particularly common in people affected by a given genetic condition and understood as an expression of the genotype. It should not be used as synonymous with a behavioural syndrome or psychiatric diagnosis. ${ }^{5}$ Behavioural phenotypes of different biologically determined mentally handicapping conditions have now been described for Angelman's syndrome, ${ }^{6}$ fragile $\mathrm{X}$ syndrome, ${ }^{7}$ tuberous sclerosis, ${ }^{8}$ and Williams syndrome. ${ }^{9}$ Most reports of Noonan's syndrome restrict their attention to their physical characteristics, developmental delay, and attainments. There are few research data available about the behavioural characteristics of children with Noonan's syndrome. Hill reports problematic peer relationships, stubbornness, and feeding difficulties in infancy. ${ }^{10}$

The identification of behavioural phenotypes has important clinical implications for diagnosis, genetic counselling, and management of children and families with genetic syndromes. The recognition that certain syndromes result in particular behavioural problems helps carers in their understanding and acceptance of their child's condition, facilitates early diagnosis, and alerts the clinician to the likely need for particular care in designing case management plans. Recent reviews of Noonan's syndrome have commented on areas of limited knowledge including difficulty in making the diagnosis, the lack of a biochemical marker, and uncertainties about recurrence risks. ${ }^{1211}$ Further knowledge of the behavioural and psychiatric characteristics of Noonan's syndrome may improve diagnosis and subsequent management.

This study describes the behavioural phenotype and psychiatric symptoms of 21 children with definite Noonan's syndrome. Data from the behavioural phenotypes questionnaire (BPQ) are presented and, in addition, we asked parents to complete a standard questionnaire of psychiatric morbidity, the child behaviour checklist (CBCL) ${ }^{12}$ for both subjects with Noonan's syndrome and a sibling control group. The study is part of a larger study delineating haematological and genetic characteristics of Noonan's syndrome. As far as we are aware, this is the first published study of both behavioural and psychiatric aspects of the syndrome.

\section{Methods}

A total of 27 patients with Noonan's syndrome between the ages of 2 and 19 years were identified from the diagnostic register, department of clinical genetics, Royal Manchester Children's Hospital (RMCH). This is a regional paediatric genetics centre and all cases had been referred for diagnostic assessment and subsequent follow up. All patients had been assessed by a consultant paediatric geneticist (MS) before the start of this study and all children easily satisfied the criteria of Allanson et al for a diagnosis of Noonan's syndrome. ${ }^{1}$ The children were invited to attend with their families for a half day's assessment at $\mathrm{RMCH}$. The first part of this assessment included a detailed clinical exami- 
Behavioural characteristics of 21 children with Noonan's syndrome (BPQ)

\begin{tabular}{lr}
\hline & No (\%) positive \\
Behavioural characteristics & responses \\
\hline Feeding & \\
Normal intake of food & $7(33)$ \\
Eating less than normal & $10(48)$ \\
Pica & $3(14)$ \\
Faddy eating & $14(67)$ \\
Sleep & \\
Regular sleep pattern & $20(95)$ \\
Social behaviour & \\
Normal interest in others & $12(57)$ \\
Overfriendly with strangers & $7(33)$ \\
Unusual eye contact & $6(29)$ \\
Facial grimacing & $7(33)$ \\
Sexualised behaviour & 0 \\
Peculiar object attachments & $5(24)$ \\
Liking of routine, obsessional behaviour & $9(43)$ \\
Communication & $11(52)$ \\
Echolalia & \\
Behaviour/activity & $5(24)$ \\
Underactive & $10(48)$ \\
Normally active & $6(28)$ \\
Overactive & $15(71)$ \\
Clumsiness & $13(62)$ \\
Squirmy/fidgety & $5(24)$ \\
Self injurious behaviours & \\
Aggressive behaviour & $6(29)$ \\
Adults & $4(19)$ \\
Children & $13(62)$ \\
Stubborn & $11(52)$ \\
Mood & $11(52)$ \\
Difficult to interpret mood & $5(24)$ \\
Excessively happy & $4(19)$ \\
Unhappy & $9(42)$ \\
Often tearful & $9(42)$ \\
Sritable & \\
\hline & \\
\hline & \\
\hline &
\end{tabular}

nation and haematological studies. These results will be presented separately (A Massarano et al; unpublished). The BPQ was administered as part of a semistructured interview with parents and the first author. Finally, parents were asked to complete the CBCL for their child and a maximum of two siblings closest in age and sex to the child with Noonan's syndrome.

The BPQ is a parent report questionnaire that can be administered as a postal questionnaire or as a semistructured interview. It has been developed specifically for the purpose of obtaining cross syndrome data ${ }^{4}$ and contains questions on diagnosis, physical aspects, prenatal problems, developmental items, and behaviour.

The CBCL is used to obtain standardised parental reports of children's problem behaviours aged 4-16 years. The CBCL consists of 20 competence items and 120 behaviour problem items scored on a three point scale. Only the findings from the problem behaviour section will be reported. A total problem score is computed by summing all scores. In addition behavioural items are subdivided to form eight 'cross informant syndromes', namely: withdrawal, somatic problems, anxious/ depressed, social problems, thought problems, attention problems, delinquent behaviour, and aggressive behaviour. A child is scored on the above scales according to CBCL norms for age and sex. We shall refer to children scoring above the recommended cut off scores overall and for the eight syndrome scales as 'cases'.

\section{Results}

Twenty one children with Noonan's syndrome were assessed from 18 families. Eighteen children attended for interview and a further three subjects completed postal questionnaires. Ages ranged from $2 \cdot 6$ years to $16 \cdot 4$ years with a median age of 9.3 years. There were 14 males and seven females. A diagnosis of Noonan's syndrome was confirmed by MS in all cases and one child had a diagnosis of athetoid cerebral palsy in addition to Noonan's syndrome. BPQ data were collected for 21 children with Noonan's syndrome; CBCL data were available for 19 children with Noonan's syndrome and 10 siblings without the syndrome.

\section{BEHAVIOURAL PHENOTYPES QUESTIONNAIRE Non-behavioural aspects}

Data from the BPQ showed the group to be fairly competent overall. Clinical details agreed in general with other reports (A Massarano et al; unpublished). Prenatal problems were common, with $11(52 \%)$ describing a complication of pregnancy and $11(52 \%)$ requiring admission to the special care baby unit. A normal vaginal delivery occurred in $15(71 \%)$. Short stature was present in $15(71 \%)$, abnormal vision in $12(57 \%)$, and hearing was impaired in three $(14 \%)$. The majority of children were competent in fine and gross motor skills. More than $75 \%$ could feed and dress themselves at the time of assessment. Nine $(43 \%)$ showed some language impairment. Mean age at diagnosis (5.0 years) was younger than that described by Sharland et al. ${ }^{11}$ Twelve of the 18 school aged children attended mainstream education.

\section{BEHAVIOURAL ASPECTS}

Findings for the behavioural domain of the BPQ for the 21 subjects with Noonan's syndrome are presented in the table. No behavioural characteristic was present in over $75 \%$ of subjects. The following eight characteristics were described for more than $50 \%$ of the group: clumsiness 15 (71\%), faddy eating 14 $(67 \%)$, squirmy/fidgety $13(62 \%)$, stubborn 13 $(62 \%)$, echolalia $11(52 \%)$, difficult to interpret mood $11(52 \%)$, excessively happy 11 $(52 \%)$, and irritable $11(52 \%)$.

\section{CHILD BEHAVIOUR CHECKLIST}

Psychiatric findings: on the basis of the overall CBCL score, nine $(47 \%)$ of the subjects with Noonan's syndrome scored above the cut off as a reported case. Of the sibling controls, two scored as cases. One of these was a sibling of a case of Noonan's syndrome and the other was not. The proportion of subjects with Noonan's syndrome scoring as cases on the CBCL compared with controls failed to attain statistical significance $\left(\chi^{2}=2 \cdot 43, p=0 \cdot 12\right)$.

The mean (SE) overall CBCL score for subjects with Noonan's syndrome was $41.9(5 \cdot 2)$ and for controls $27 \cdot 0 \quad(8 \cdot 2)$. Differences between mean score on the CBCL for those with Noonan's syndrome and controls failed to attain statistical significance using the Mann-Whitney test $(\mathrm{W}=112 \cdot 0, \mathrm{p}=0.0812)$. 
Examining the eight syndrome scales of the CBCL, social problems (six cases $(32 \%)$ ) and attention problems (five cases $(26 \%)$ ) were noted for those with Noonan's syndrome. Differences between Noonan's syndrome subject subscale scores and sibling control scores did not attain statistical significance.

\section{Discussion}

This is the first published study of behavioural and psychiatric characteristics of children with Noonan's syndrome. Although there are no agreed criteria for defining a behavioural phenotype of a given condition, ${ }^{13}$ our results highlight certain characteristics that were present in greater than $50 \%$ of a sample of children with definite Noonan's syndrome. They suggest a behavioural profile for Noonan's syndrome. Stubbornness, clumsiness, mood problems, communication problems, and faddy eating were described for more than half of our sample. Notably few children showed autistic features, sleep difficulties, severe aggression, sexualised behaviours, or anxiety. These findings are in agreement with the pilot study of Hill. ${ }^{10}$ Results from the measure of parent reported psychiatric symptomatology agree with these findings, outlining problems on two scales of the CBCL: social relationships and attention deficit. For the remaining six scales of the CBCL (withdrawn, somatic complaints, anxious/depressed, thought problems, delinquent behaviour, aggressive behaviour) few problems are reported. This is a small sample and, as yet, there are no comparison data for the $\mathrm{BPQ}$.

A number of factors other than genotype will be contributing to any describable patterns of behaviour in children with Noonan's syndrome such as parenting styles and psychosocial stresses within the family. Noonan's syndrome is a disorder with important medical implications and the diagnosis may have a significant psychological impact on families. Receiving a diagnosis of Noonan's syndrome may influence parent-child relationships and secondarily, behaviour. Studying non-affected siblings within the same family has been used as a way of controlling for intrafamilial sources of behavioural symptoms. We found a higher rate of parent reported behavioural problems in children with the syndrome compared with sibling controls. In our small study, this difference failed to reach statistical significance. We note that the rate of 'caseness' on the CBCL in the Noonan's syndrome group was nearly $50 \%$ whereas in general population studies, the rate is usually of the order of $15 \% .^{14}$ The psychiatric symptoms described for children with Noonan's syndrome are non-specific and likely to have complex multiple aetiological factors. This finding may highlight a need for specialist provision for some families. We hope that this knowledge about behavioural and psychiatric aspects of Noonan's syndrome may contribute to diagnosis and subsequent management of children and families as well as acting as core data for comparison with other genetic syndromes.

We wish to thank secretarial staff in the department of genetics, staff nurse Carolyn Lathan, and all of the children and families who participated in our study.

Full results of the BPQ and CBCL are available on request from the first author.

1 Allanson JE, Hall JG, Hughes HE, Preus M, Witt RD. Noonan syndrome: the changing phenotype. $A m$ f Med Genet 1985; 21: 507-14

2 Mendez HM, Opitz J. Noonan syndrome: a review. $A m \mathcal{F}$ Med Genet 1985; 21: 493-506.

3 Money J, Kalus ME. Noonan's syndrome. IQ and specific disabilities. Am F Dis Child 1979; 133: 846-50.

4 O'Brien G. Behavioural phenotypy in developmental psychiatry. European Child and Adolescent psychiatry 1992; psychiatry. Europ $1: 1-61$.

5 O'Brien G. Behavioural phenotypes and their measurement. Dev Med Child Neurol 1992; 34: 365-7.

6 Clayton-Smith J. Behaviour in Angelman syndrome. Behavioural phenotypes: abstracts and syndrome information. Oxford: SSBP Publications, 1990.

7 Borghgraef M, Fryns JP, Van den Bergh R, Pyck K, Van den Berghe V. Pre and post pubertal fragile X males: a study of the intelligence and psychological profile of 17 fragile $X$ boys. In: Fraser WI, ed. Key issues in mental retardation research. London: Routledge, 1990: 94-108.

8 Hunt A, Dennis J. Psychiatric disorder among children with tuberous sclerosis. Dev Med Child Neurol 1987; 29: 190-8.

9 Udwin O, Yule W, Martin N. Cognitive abilities and behavioural characteristics of children with idiopathic

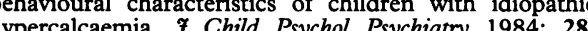
hypercalca $297-309$.

10 Hill P. Noonan syndrome. Behavioural phenotypes: abstracts and syndrome information. Oxford: SSBP Publications, and symd

11 Sharland M, Burch M, McKenna WM, Paton MA. A clinical study of Noonan syndrome. Arch Dis Child 1992 67: 178-83.

12 Achenbach TM, Edelbrook C. Manual for the child behaviou checklist and revised child behaviour profile. Burlington VT, University of Vermont: Department of Psychiatry, 1983.

13 Flint J, Yule W. Behavioural phenotypes. In: Rutter $M$ Taylor E, Hersov L, eds. Child and adolescent psychiatry. Modern approaches. Oxford: Blackwell Scientific Publications, 1994: 666-87.

14 Verhulst FC, Koot HM. Child psychiatric epidemiology: con cepts, methods, and findings. London: Sage, 1992. 